\title{
Intradermal Injection of Capsaicin in Humans Produces Degeneration and Subsequent Reinnervation of Epidermal Nerve Fibers: Correlation with Sensory Function
}

\author{
Donald A. Simone, ${ }^{1,2}$ Maria Nolano, ${ }^{3}$ Timothy Johnson, ${ }^{4}$ Gwen Wendelschafer-Crabb, ${ }^{4}$ and \\ William R. Kennedy ${ }^{4}$ \\ Departments of ${ }^{1}$ Psychiatry, ${ }^{2}$ Preventive Sciences, and ${ }^{4}$ Neurology, University of Minnesota, Minneapolis, Minnesota \\ 55455, and ${ }^{3}$ Salvatore Maugeri Foundation, Campoli M.T. (BN), Italy
}

The ability of capsaicin to excite and subsequently to desensitize a select group of small sensory neurons has made it a useful tool to study their function. For this reason, application of capsaicin to the skin has been used for a variety of painful syndromes. We examined whether intradermal injection of capsaicin produced morphological changes in cutaneous nerve fibers that would account for its analgesic properties by comparing cutaneous innervation in capsaicin-treated skin with psychophysical measures of sensation. At various times after capsaicin injection, nerve fibers were visualized immunohistochemically in skin biopsies and were quantified. In normal skin the epidermis is heavily innervated by nerve fibers immunoreactive for protein gene product (PGP) 9.5, whereas fibers immunoreactive for substance $P(S P)$ and calcitonin gene-related peptide (CGRP) are typically associated with blood vessels. There was nearly complete degeneration of epidermal nerve fibers and the subepidermal neural plexus in capsaicin-treated skin, as indicated by the loss of immunoreactivity for PGP 9.5 and CGRP. The effect of capsaicin on dermal nerve fibers immunoreactive for SP was less obvious. Capsaicin decreased sensitivity to pain produced by sharp mechanical stimuli and nearly eliminated heat-evoked pain within the injected area. Limited reinnervation of the epidermis and partial return of sensation occurred 3 weeks after treatment; reinnervation of the epidermis was $\sim 25 \%$ of normal, and sensation improved to $50-75 \%$ of normal. These data show that sensory dysfunction after capsaicin application to the skin results from rapid degeneration of intracutaneous nerve fibers.

Key words: pain; analgesia; protein gene product 9.5; intracutaneous nerves; immunohistochemistry; confocal microscopy
Capsaicin, the active pungent ingredient in hot peppers, is a unique tool used to study the functions of a subset of sensory neurons, including nociceptive neurons. Early studies focused on the neurotoxic actions of capsaicin applied systemically in high doses to neonatal or adult rats (for review, see Nagy, 1982; Fitzgerald, 1983; Russell and Burchiel, 1984; Buck and Burks, 1986; Holzer, 1991). It was found that capsaicin destroys a subset of small diameter primary afferent fibers and their cell bodies.

Topical application of capsaicin evokes burning pain, neurogenic inflammation (vasodilatation and plasma extravasation), and hyperalgesia to heat and mechanical stimuli (Szolcsányi, 1977; Carpenter and Lynn, 1981; Culp et al., 1989; Simone and Ochoa, 1991). After repeated applications, the treated area becomes less sensitive to pain. This desensitizing action has made capsaicin attractive for use as a peripherally acting analgesic for chronic painful syndromes (Capsaicin Study Group, 1991; Fusco and Giacovazzo, 1997).

Intradermal injection of capsaicin quickly deposits a quantified amount directly into human skin. This produces a sensation of intense burning pain and hyperalgesia to heat and mechanical

Received May 13, 1998; revised Aug. 12, 1998; accepted Aug. 13, 1998.

This work was supported in part by National Institutes of Health Grants NS31223 (D.A.S.) and NS31397 (W.R.K.) and by a grant from Toray Industries Inc. (W.R.K.). We thank Dr. Paul Thuras for assistance with statistical analyses.

Correspondence should be addressed to Dr. Donald A. Simone, Department of Psychiatry, University of Minnesota, 420 Delaware Street SE, Box 392, Minneapolis, MN 55455.

Copyright (ㄷ) 1998 Society for Neuroscience $\quad 0270-6474 / 98 / 188947-08 \$ 05.00 / 0$ stimuli (Simone et al., 1987, 1989; LaMotte et al., 1991, 1992), followed by a rapid desensitization characterized by diminished pain sensation at the site of application (LaMotte et al., 1991). Electrophysiological studies have shown that shortly after intradermal injection of capsaicin, C-fiber polymodal nociceptors can become insensitive to mechanical and heat stimuli (Baumann et al., 1991). Furthermore, this effect of capsaicin is well localized to the injection site because only the portion of the receptive field exposed to capsaicin becomes desensitized. Thus, diminished pain sensation at the site of capsaicin injection is attributed to desensitization of nociceptors.

The mechanisms underlying rapid desensitization and hypalgesia after local capsaicin application in humans are unclear. Desensitization of capsaicin-sensitive afferent fibers involves a continuum of physiological and morphological changes that are dependent on capsaicin dose and route of administration. The effects of capsaicin on neural function, whether applied systemically or locally, have been categorized into various stages in animal studies and range from conduction block with reversible ultrastructural changes in peripheral nociceptive endings to irreversible degeneration of nociceptive neurons and their processes (Szolcsányi, 1993). For example, although systemic application of high doses of capsaicin destroys certain sensory neurons, capsaicin applied to the peripheral nerve endings in the cornea produces swelling of mitochondria and a reduction in the number of microvesicles in unmyelinated nerve endings without evidence of axonal degeneration (Szolcsányi et al., 1975). Morphological correlates of functional desensitization after capsaicin application to 
skin are unknown. Although topical capsaicin decreased the number of nerve fibers in the epidermis as observed in a blister roof (Reilly et al., 1997), this was not verified by skin biopsy or sensory testing. Therefore, in this correlative study in humans, psychophysical measures of cutaneous sensation and immunohistochemical techniques were used to determine whether the hypalgesia after intradermal injection of capsaicin could be attributed to morphological changes in epidermal nerve fibers (ENFs).

A preliminary report has been published previously (Simone et al., 1996).

\section{MATERIALS AND METHODS}

Subjects. Eight subjects (six male and two female) ranging in age from 24 to 69 years participated. Each subject provided informed consent to a protocol that was approved by the Institutional Review Board Human Subjects Committee of the University of Minnesota.

Intradermal injection of capsaicin. Capsaicin was dissolved in a vehicle containing $7.5 \%$ Tween 80 in saline as described previously (Simone et al., 1987, 1989; LaMotte et al., 1991). All injections were given into test areas $(5 \mathrm{~mm}$ in diameter) marked on the lateral aspect of the upper arm. Capsaicin doses of either $0.2,2$, or $20 \mu \mathrm{g}$ in a volume of $20 \mu \mathrm{l}$ or an equal amount of the vehicle was injected into each site using a $0.5 \mathrm{ml}$ insulin syringe. A maximum of seven injections were given into each shoulder. Before each injection, the skin was anesthetized with an intradermal injection of $1 \%$ lidocaine $(0.3-0.5 \mathrm{ml})$.

Psychophysical measures of cutaneous sensation. Heat pain, pricking pain, cold sensation, and tactile threshold were evaluated within each 5 -mm-diameter test site. Thermal stimuli of $5 \mathrm{sec}$ duration were applied via a 2 -mm-diameter contact probe maintained at $53^{\circ} \mathrm{C}$ for heat pain or $1^{\circ} \mathrm{C}$ for cold sensation. Subjects judged the magnitude of heat pain and the magnitude of cold sensation using a visual analog scale ranging from 0 (no pain) to 10 (most severe pain imaginable). Heat and cold stimuli were each applied five times, and the mean magnitude of pain and cold sensation was determined. Pricking pain was evoked by a sharp probe (50- $\mu \mathrm{m}$-diameter tip) attached to a nylon monofilament with a bending force of $95 \mathrm{mN}$. This probe did not penetrate the skin. The stimulus was applied 10 times, each for a duration of 1-2 sec. The proportion of stimulus presentations that evoked pain, as well as the magnitude of pain, was recorded. Tactile threshold (in $\mathrm{mN}$ ) was determined by the use of calibrated Semmes-Weinstein monofilaments. Threshold was defined as the smallest monofilament that could be perceived at least $50 \%$ of the time. Individual monofilaments were applied 10 times beginning with a suprathreshold stimulus. All sensory tests were performed at each test site before and at various times after injection.

Skin biopsy and immunohistochemistry. Skin biopsies were obtained from vehicle- and capsaicin-treated sites and occasionally from untreated skin. After the skin was anesthetized by intradermal injection of $1 \%$ xylocaine (Astra, Westborough, MA), the biopsy was made with a $3 \mathrm{~mm}$ punch tool (Acupunch; Acuderm, Fort Lauderdale, FL) and processed as described previously (Kennedy et al., 1996). Briefly, biopsies were fixed in Zamboni's solution, cryoprotected, and sectioned with a freezing sliding microtome (Leica, Nussloch, Germany). Diluent and washing solutions comprised $1 \%$ normal donkey serum (Jackson ImmunoResearch, West Grove, PA) in 0.1 м PBS with $0.3 \%$ Triton X-100 (Sigma, St. Louis, MO). Floating sections were blocked with $5 \%$ normal donkey serum in the diluent solution. Nerve and tissue antigens were localized using primary antibodies to protein gene product (PGP) 9.5 (1:800; Ultraclone, Isle of Wight, England), substance P (SP) (1:1000; Incstar, Stillwater, MN), calcitonin gene-related peptide (CGRP) (1:1000; Amersham, Arlington Heights, IL) and type IV collagen (Chemicon, Temecula, CA), each diluted in PBS-Triton X-100-NGS. Nonimmune serum was used for negative controls. Secondary antibodies specific to the $\operatorname{IgG}$ species used as a primary antibody and labeled with cyanine dye fluorophores 3.18 and 5.18 (Jackson ImmunoResearch) were used to locate two antigens in each section. After immunohistochemical processing, sections were adhered to coverslips with agar, dehydrated via an alcohol series, cleared with methyl salicylate, and mounted in DPX (Fluka BioChemika, Ronkonkoma, NY).

Imaging and quantification of ENFs. Images of sections that were double stained with PGP 9.5 and type IV collagen were collected with a laser-scanning confocal microscope (Bio-Rad, Hercules, CA) with a Nikon $20 \times$ planapochromate objective (numerical aperture, 0.75) and appropriate filters. Each image set comprised a $z$-series that was acquired in $2 \mu \mathrm{m}$ increments throughout the thickness of the section.

Quantitative analyses of ENFs were performed as described previously (Kennedy et al., 1996). Briefly, $z$-series image stacks of PGP 9.5-immunostained ENFs were acquired from the biopsy sections with the confocal microscope, and the images were analyzed with Neurolucida software (MicroBrightField, Colchester, VT) by tracing nerve fibers in three dimensions. Individual ENFs are counted as they pass through the basement membrane. Branching occurring within the epidermis did not increase the number of ENFs counted. Epidermal nerve counts of PGP 9.5-immunoreactive fibers were standardized for section thickness (30 $\mu \mathrm{m})$ and expressed as the number of fibers per millimeter of epidermis.

The subepidermal neural plexus and SP- and CGRP-immunoreactive fibers were examined qualitatively by visual inspection with the use of fluorescence microscopy.

Data analyses. ENFs were counted, quantified, and compared with the number of ENFs per millimeter length in normal epidermis. A one-way ANOVA was used to compare the number of ENFs present $3 \mathrm{~d}$ after intradermal injection of vehicle and of $0.2,2$, and $20 \mu \mathrm{g}$ doses of capsaicin. The innervation of epidermis before and between 1 and 4 weeks after an intradermal injection of $20 \mu \mathrm{g}$ was assessed by repeatedmeasures ANOVA. Comparisons were made between the number of ENFs in capsaicin-treated skin, vehicle-treated skin, and normal untreated skin.

The effect of capsaicin on psychophysical measures of heat pain sensation, cold sensation, and tactile sensation and on the proportion of sharp stimuli perceived as painful was assessed using ANOVAs with repeated measures. Separate analyses were used to examine the effect of capsaicin dose on the various sensory modalities and to evaluate changes in sensation over a 4 week time period after a single injection of $20 \mu \mathrm{g}$ of capsaicin.

Experimental design. To determine the effect of graded doses of capsaicin on the sensation and morphology of ENFs, we gave each of five subjects one set of four intradermal injections on each upper arm. A set of injections consisted of capsaicin doses of $0.2,2$, and $20 \mu \mathrm{g}$ and the vehicle. Sensation was assessed and skin biopsy was performed at each injection site after $24 \mathrm{hr}$ for one set of injections and after $72 \mathrm{hr}$ for the other set.

In a separate experiment, we determined the time course and extent of reinnervation and whether reinnervation is accompanied by the return of normal pain sensation. Five subjects were given four intradermal injections of $20 \mu \mathrm{g}$ of capsaicin into the upper arm. Evoked sensation and cutaneous innervation were assessed at each injection site at 1,2,3, or 4 weeks after injection. Three subjects received one additional injection of capsaicin, and measurements were also made at 6 weeks after injection.

\section{RESULTS}

\section{Effect of capsaicin on the number of ENFs and cutaneous sensation: dose-response relationships}

In vehicle-treated skin, like normal untreated skin, PGP 9.5immunoreactive nerve fibers are abundant in the subepidermal neural plexus, which lies just below the basement membrane (Fig. $1, V e h)$. The epidermis is richly innervated by fibers that originate in the subepidermal plexus and project up through the basement membrane and terminate in the epidermis. It is likely that all nerve fibers have been visualized because PGP 9.5immunoreactive axons are greater in number and density and the staining is stronger than that seen with antisera to other neural markers (Karanth et al., 1991). It has been shown previously that nerve fibers extending into the epidermis are unmyelinated (Ochoa, 1984; Wang et al., 1990; Kennedy and WendelschaferCrabb, 1993).

Capsaicin produced a rapid, dose-dependent degeneration of intracutaneous nerve fibers and a dramatic decrease in the sensation of pain produced by heat and mechanical stimuli. All three doses of capsaicin caused a significant reduction in the mean number of ENFs as compared with that in vehicle-treated skin $(p<0.05)$. Although nerve degeneration was evident at $24 \mathrm{hr}$ after injection, the magnitude and spatial extent of fiber loss were more pronounced at $72 \mathrm{hr}$ after injection. An example of nerve 

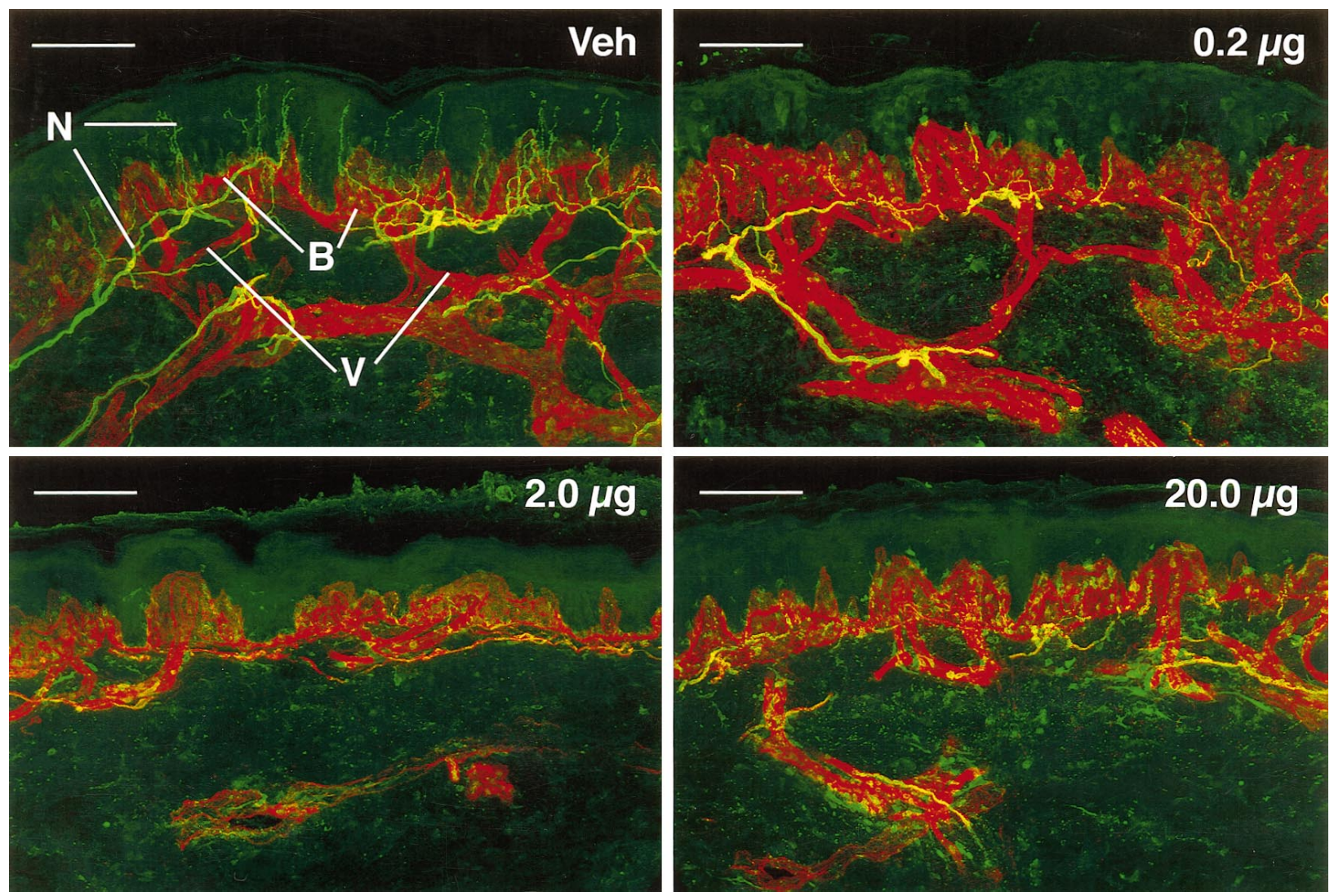

Figure 1. Confocal images showing innervation of epidermis and superficial dermis for one subject at $72 \mathrm{hr}$ after a single injection of vehicle (Veh) or capsaicin doses of $0.2,2.0$, or $20 \mu \mathrm{g}$. Nerve fibers $(N)$ immunoreactive for PGP 9.5 appear yellow-green; the basement membrane $(B)$ and vessels $(V)$ appear red. Scale bars, $100 \mu \mathrm{m}$.

degeneration after capsaicin injection is provided in Figure 1, which shows confocal images of skin biopsies for one subject with typical neural degeneration observed at $72 \mathrm{hr}$ after injection. After the lowest dose of capsaicin, loss of PGP 9.5immunoreactive nerve fibers was restricted primarily to fibers located in the epidermis, with little to moderate disruption of nerve fibers in the subepidermal neural plexus. Higher doses of capsaicin resulted in complete loss of PGP 9.5-immunoreactive ENFs plus various degrees of disruption or complete loss of the subepidermal nerve plexus.

Nerve fibers immunoreactive for CGRP and SP are sparsely distributed throughout the papillary dermis where they are typically associated with capillary loops. CGRP-immunoreactive nerve fibers occasionally penetrate the epidermis, whereas SPimmunoreactive fibers are sparse in the subepidermal neural plexus and rarely enter the epidermis. Complete loss of CGRPimmunoreactive fibers was also observed $72 \mathrm{hr}$ after capsaicin injection. The effect of capsaicin on SP-immunoreactive nerve fibers was difficult to assess because very few fibers are normally found in the superficial dermis, and after capsaicin a few SPimmunoreactive nerve fibers were found in some subjects, whereas no SP-immunoreactive fibers were found in other subjects.

The relationship between capsaicin dose, somatic sensation, and the number of PGP 9.5-immunoreactive ENFs at $72 \mathrm{hr}$ after injection is summarized for all five subjects in Figure 2. The mean ( \pm SEM) decrease in the number of ENFs per length (in millimeters) of epidermis at $72 \mathrm{hr}$ after injection of $0.2,2$, and $20 \mu \mathrm{g}$ of capsaicin was $43.5 \pm 13.2,98.7 \pm 1.33$, and $99.9 \pm 0.99 \%$,

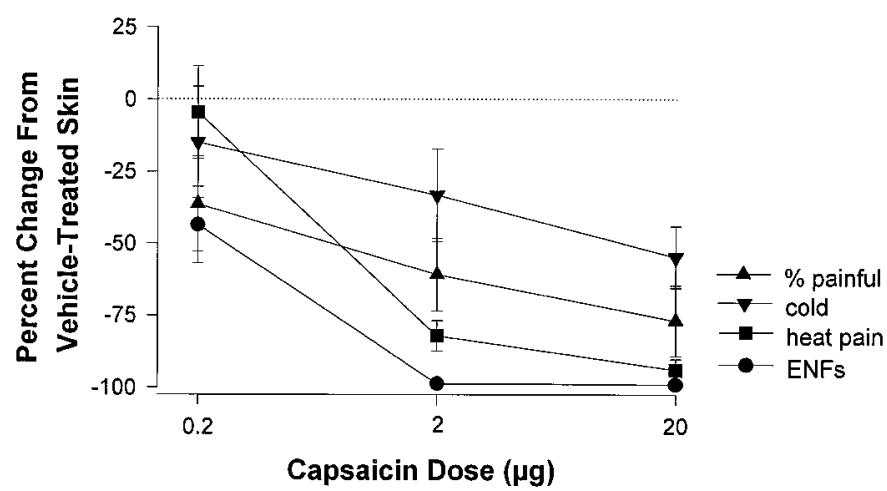

Figure 2. Somatic sensation and the number of ENFs for all five subjects at $72 \mathrm{hr}$ after injection of capsaicin. Data are expressed as the mean $( \pm$ SEM) percent change from the data for vehicle-treated skin. For heat pain and cold sensation, data represent the change in the magnitude of sensation. Mechanical pain sensation is represented as the change in the proportion of stimuli perceived as painful.

respectively, compared with that in vehicle-treated skin. The decrease in the number of ENFs was associated with diminished pain sensation. One-way ANOVAs revealed that capsaicin decreased the magnitude of heat pain sensation $(p<0.001)$ and the detection of sharp pain sensation $(p<0.001)$. However, heat pain sensation was more sensitive to capsaicin treatment than was mechanical pain sensation. The magnitude of heat pain sensation decreased significantly after injection of 2 and $20 \mu \mathrm{g}$ of capsaicin $(p<0.05)$, whereas the proportion of sharp mechanical stimuli 

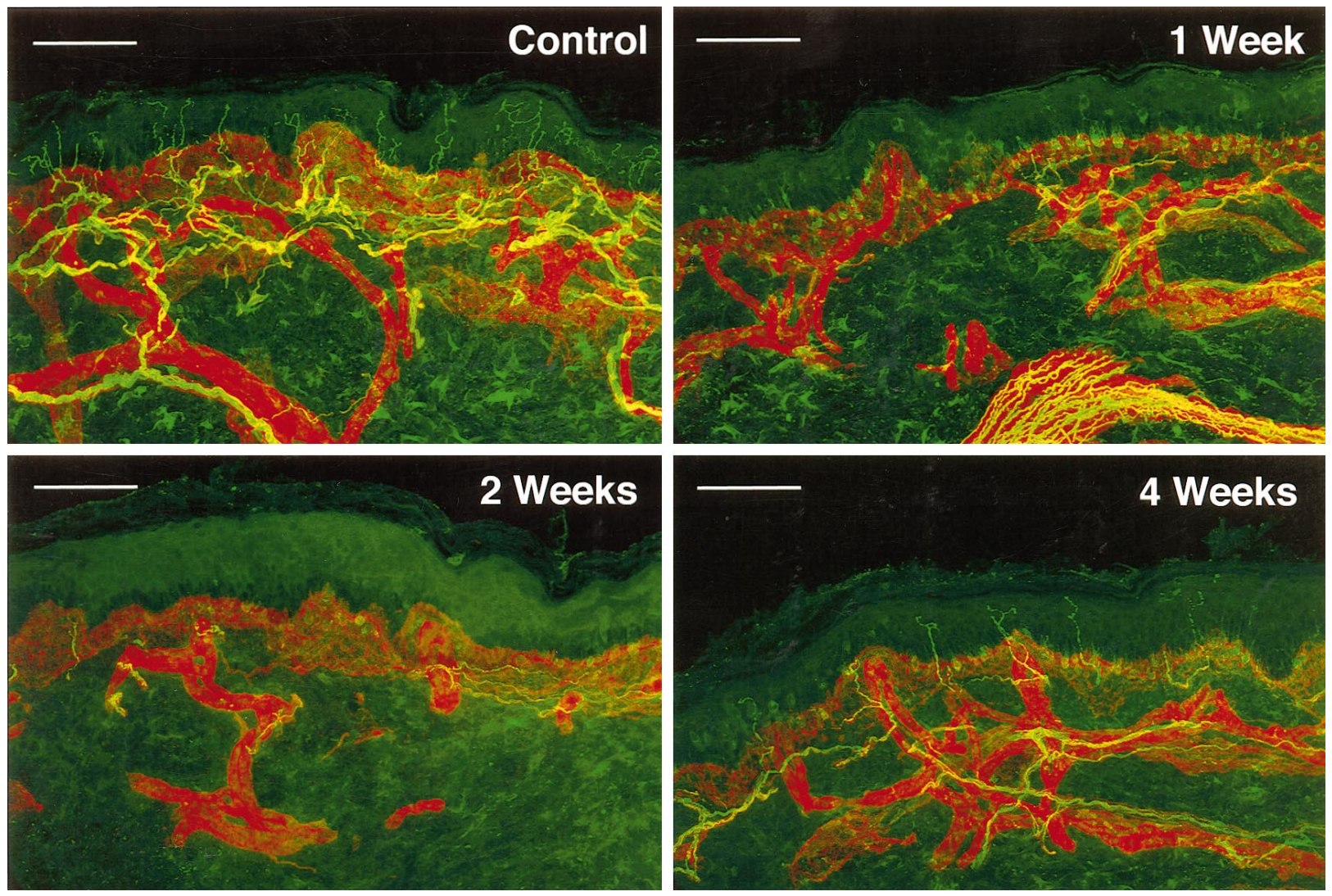

Figure 3. Confocal images showing denervation and reinnervation of epidermis and superficial dermis by PGP 9.5-immunoreactive nerve fibers for one subject. Biopsies were taken from capsaicin-treated $(20 \mu \mathrm{g})$ skin at 1,2 , and 4 weeks after injection and from normal untreated skin. The appearance of nerve fibers immunoreactive for PGP 9.5 is the same as that described in Figure 1. Scale bars, $100 \mu \mathrm{m}$.

perceived as painful decreased significantly after the $20 \mu \mathrm{g}$ dose $(p<0.05)$. Tactile threshold was not altered significantly after capsaicin injection, but the effect of capsaicin on this measure varied considerably between subjects. Although the magnitude of cold sensation did not decrease significantly, the subjective magnitude of cold decreased $>50 \%$ in two of five subjects after the 2 $\mu \mathrm{g}$ dose and in three of five subjects after the $20 \mu \mathrm{g}$ dose. The remaining subjects experienced a lesser decrement in cold sensation or no change at all. Injection of the vehicle did not produce significant changes in any evoked sensations or in innervation density as compared with that in normal untreated skin.

\section{Reinnervation of the epidermis and restoration of sensory function}

To determine the extent to which ENFs regenerated after capsaicin as well as the time course of epidermal reinnervation, we performed skin biopsies and sensory tests at capsaicin injection sites 1-4 $(n=5)$ or $6(n=3)$ weeks after the injection. A one-way ANOVA indicated a significant decrease in ENFs after capsaicin $(p<0.001)$ compared with that in normal skin. All subjects exhibited denervation in capsaicin-treated skin during the first 2 weeks after injection, and ENFs were rarely observed. Similarly, nerve fibers in the subepidermal neural plexus were also sparse during this time period. Reinnervation of the epidermis by ENFs began during the third and fourth weeks after the capsaicin injection and was characterized by the return of an intact subepidermal neural plexus and the reappearance of sparse nerve fibers in the epidermis. However, the innervation of epidermis during this time was still dramatically impaired, and the number of ENFs per length (in millimeters) of epidermis ranged from only 12 to $29 \%$ of that in normal skin. Figure 3 shows confocal images of biopsy sections stained for PGP 9.5 immunoreactivity in normal untreated skin and in skin at 1,2, and 4 weeks after capsaicin treatment for one subject. For this subject, who exhibited the most reinnervation of all subjects tested, innervation of the epidermis did not improve further between 4 and 6 weeks (we did not examine at any later time).

Nerve fibers immunoreactive for CGRP also reappeared 3-4 weeks after capsaicin (Fig. 4). These CGRP-immunoreactive nerve fibers were never observed in the epidermis or superficial dermis 1 week after capsaicin. However, they were found in the dermis, but not the epidermis, 4 weeks after injection of capsaicin. Although quantitative measures of the number of CGRPimmunoreactive fibers were not made, the number of fibers present at 4 weeks after capsaicin appeared to be less than normal, as with PGP 9.5-immunoreactive fibers.

The extent of reinnervation of SP-immunoreactive nerve fibers was difficult to assess because of the small number of fibers normally observed in the superficial dermis and epidermis. A few nerve fibers were found in at least some of the subjects at all times examined after capsaicin. These fibers were observed only in the dermis, and the number of these fibers found was similar to that in normal skin.

Gradual reinnervation of the epidermis coincided with the gradual restoration of evoked pain sensation. This is illustrated in Figure 5 that shows the mean change in the number of PGP 9.5-immunoreactive ENFs and the mean change in sensation for 

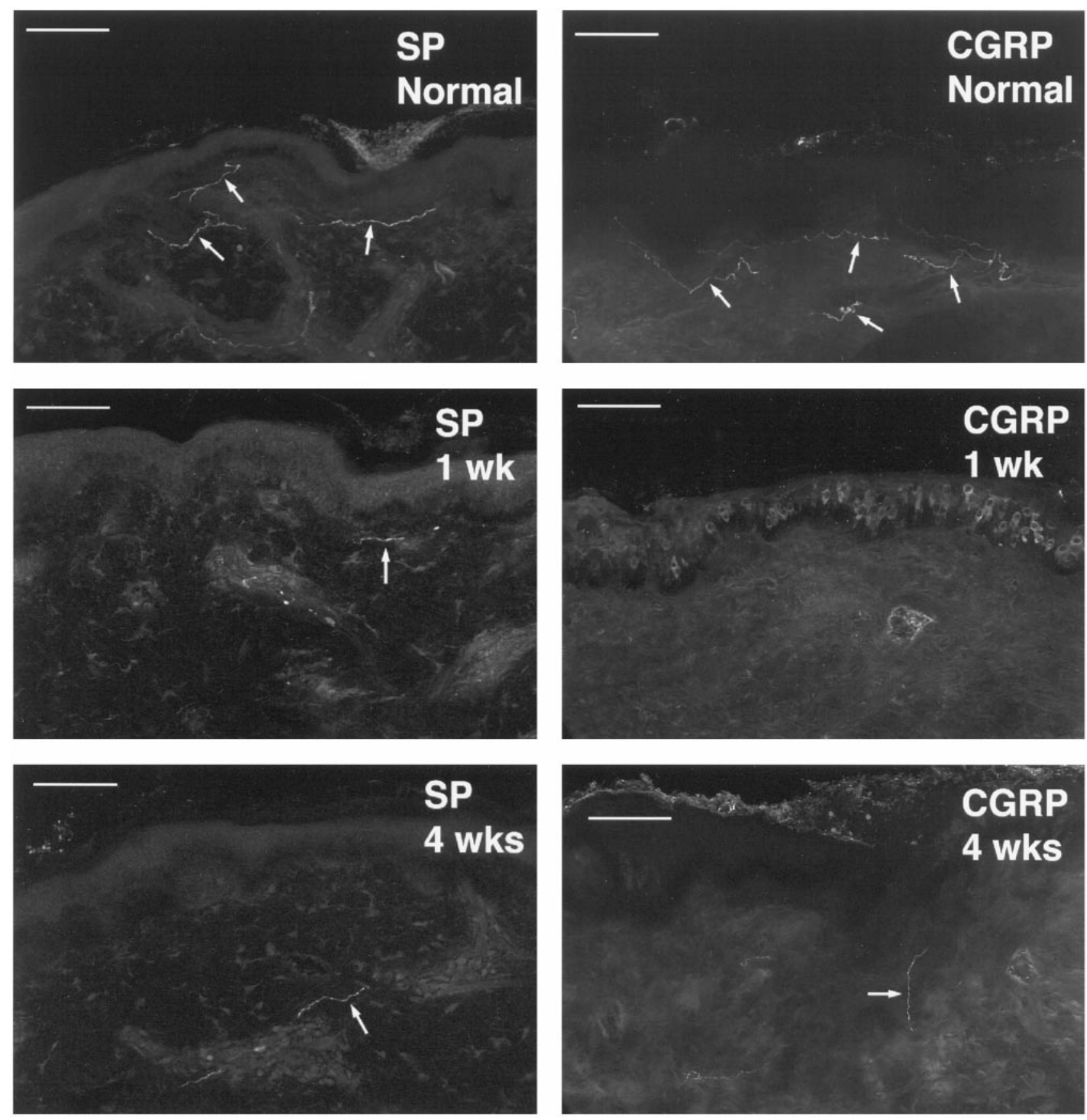

Figure 4. Confocal images of skin biopsies from one subject showing nerve fibers immunoreactive for CGRP and SP in normal skin and skin at 1 and 4 weeks after capsaicin injection. Right, CGRP-immunoreactive fibers were completely absent 1 week after capsaicin injection and reappeared 4 weeks after injection (arrows). Left, There was not a complete loss of SP-immunoreactive fibers at 1 week after capsaicin injection, and one fiber can be seen oriented horizontally below the basement membrane (arrow). At 4 weeks after capsaicin injection, fibers were occasionally found deep in the dermis (arrow) and oriented vertically toward the basement membrane. Scale bars, $100 \mu \mathrm{m}$.

all subjects. The magnitude of heat pain sensation and the percent of mechanical stimuli perceived as painful decreased significantly after capsaicin $(p<0.001)$. During the first 2 weeks after capsaicin injection, heat pain sensation was nearly eliminated, and subjects exhibited an $\sim 65 \%$ decrease in the proportion of sharp mechanical stimuli perceived as painful. However, the decrease in mechanically evoked pain sensation was variable compared with the changes in heat pain sensation. For example, during the first 2 weeks after capsaicin, three of the six subjects did not perceive sharp pain, whereas the detection of sharp pain was not altered in one subject. Although there were no significant alterations in the sensation of cold during the first 2 weeks after capsaicin, all but one subject exhibited at least a $16 \%$ decrease in the magnitude of cold sensation. Similarly, there was no significant change in tactile threshold during this time. Tactile thresholds were increased in three of the six subjects and were unchanged in the remaining three subjects.

At 3 and 4 weeks after capsaicin, detection of heat pain and of pricking pain sensation had improved and was consistent with the onset of reinnervation of the epidermis. There remained a $37 \pm$ 


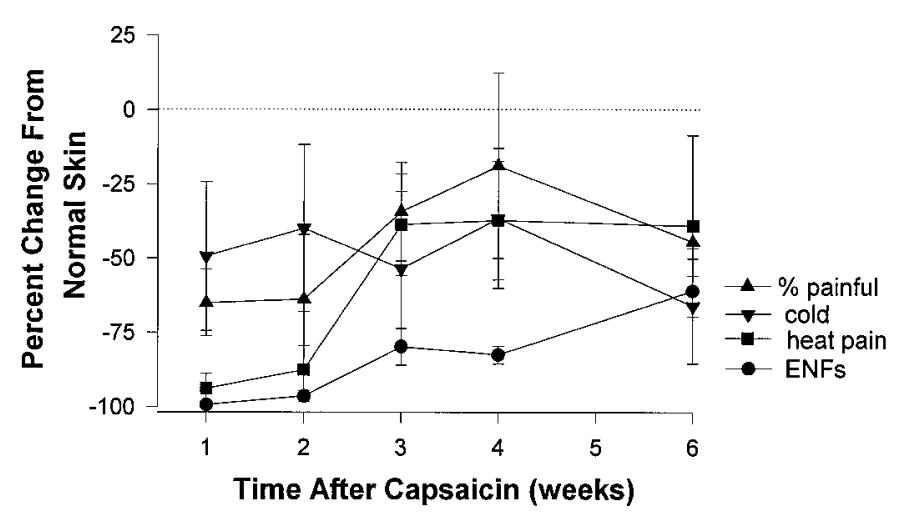

Figure 5. The mean $( \pm$ SEM) change in sensation and in the number of ENFs for all subjects through 1-6 weeks after injection of $20 \mu \mathrm{g}$ of capsaicin. Data are presented as the percent change from normal untreated skin.

$19.9 \%$ decrease in heat pain at 4 weeks after capsaicin as compared with that in normal skin. Similarly, the sensation of pricking pain also improved but was more variable than was the pain sensation evoked by heat.

\section{Localization of capsaicin-evoked nerve degeneration}

To determine the extent to which capsaicin diffused from the injection site to cause degeneration of nerve fibers adjacent to the injection site, we made one biopsy $72 \mathrm{hr}$ after injection of $20 \mu \mathrm{g}$ of capsaicin that included part of the injection site (as defined by the appearance of the bleb) and adjacent skin. A confocal image of this biopsy is provided in Figure 6. It can be seen that the left portion of the biopsied skin does not contain ENFs, whereas ENFs are clearly seen in the right portion. The right portion of the biopsy, which has a normal appearance and number of ENFs, was 1-2 $\mathrm{mm}$ from the edge of the capsaicin injection. This demonstrates that capsaicin diffuses minimally from the injection site and that nerve degeneration is restricted to the capsaicin-treated area.

\section{DISCUSSION}

The present findings demonstrate that intradermal injection of capsaicin produces rapid degeneration of nerve fibers in the epidermis and superficial dermis. This phenomenon is local in that it occurred only at the site of injection and presumably only to those nerve fibers that came in contact with capsaicin. We used loss of immunoreactivity for PGP 9.5 as evidence of degeneration. It could be argued that capsaicin interferes with expression of PGP 9.5 without producing nerve fiber destruction. However, we believe the initial loss and subsequent reappearance of immunoreactivity for PGP 9.5 coincides with degeneration and subsequent regeneration for the following reasons. First, capsaicin produced a clear disruption of the subepidermal neural plexus within $3 \mathrm{~d}$ after intradermal injection, followed by a gradual loss within the next 2 weeks. Second, loss of immunoreactivity also occurred for the neuropeptide CGRP, and there appeared to be a decrease in the number of SP-immunoreactive fibers. Third, the reappearance of immunoreactivity for PGP 9.5 was gradual and consistent with the gradual regeneration of the subepidermal neural plexus and reinnervation of the epidermis. Fourth, the number of PGP 9.5-immunoreactive fibers that reappeared was less than that in normal skin, even at 4 and 6 weeks after capsaicin injection. Fifth, the loss and subsequent reappearance of immunoreactivity for PGP 9.5 correlated well with the loss and recov- ery of somatic sensation. The combined disappearance of immunoreactivity for a cytoplasmic protein and two neuropeptides and the associated decrement in sensation strongly suggest that intracutaneous nerve fibers degenerated.

Degeneration of intradermal and epidermal nerve fibers by locally applied capsaicin raises several important issues regarding (1) the mechanisms of hypalgesia produced by capsaicin, (2) the mechanisms by which capsaicin produces local degeneration of nerve fibers, and (3) the function of epidermal nerve fibers in sensation, which are each discussed below. It is noteworthy that capsaicin produced degeneration of ENFs in the presence of local anesthesia. This suggests that degeneration produced by capsaicin is not dependent on excitation and generation of action potentials.

\section{Hypalgesia and degeneration after capsaicin}

Many studies have documented that application of capsaicin to the skin initially produces pain and hyperalgesia followed by diminished pain sensation, referred to as functional desensitization. The peripheral neural mechanisms that contribute to the positive sensory phenomena of pain and hyperalgesia produced by intradermal injection of capsaicin include excitation and sensitization of $\mathrm{C}$ polymodal nociceptors, whereas the neural mechanisms underlying functional desensitization subsequent to capsaicin treatment are unclear. One possibility is that capsaicin depletes C-fibers of neuropeptides, such as SP and CGRP, resulting in desensitization of nociceptors. Capsaicin has been shown to release these and other peptides from the peripheral endings of primary afferent fibers (Holzer, 1988; Maggi and Meli, 1988; Saria et al., 1988). Recent electrophysiological studies, however, suggest that initial desensitization and hypalgesia are related to the effects of capsaicin on neuronal ion channels. It has been found that capsaicin initially excites nociceptors by interacting with a specific receptor (Szallasi and Blumberg, 1990a,b; Caterina et al., 1997) to decrease the input resistance (Heyman and Rang, 1985), to evoke an inward current (Bevan and Docherty, 1993), and to open nonselective cation channels (Wood et al., 1988; Bleakman et al., 1990; Docherty et al., 1991). This is followed by the inactivation of voltage-gated ion channels that prevents the generation of action potentials and may account for short-lasting desensitization and hypalgesia. It is therefore possible that capsaicin interferes with the generation of action potentials by causing ultrastructural damage (e.g., mitochondrial swelling) to nociceptive endings as a result of prolonged opening of cation channels. Although the mechanisms described above may account for initial desensitization and corresponding hypalgesia, it is unlikely that they account for the long-lasting hypalgesia observed in the present study. Rather, our findings demonstrate that hypalgesia results from the loss of nerve fibers. This is the first study to examine the morphology of intradermal and ENFs located at the site of capsaicin injection. Our results are compatible with a previous study in which repeated application of topical capsaicin to the rat hindpaw produced no evidence of either nerve damage in the sciatic nerve (proximal to capsaicin application) or neuron loss in the dorsal root ganglion (DRG) (McMahon et al., 1991). Although topical capsaicin did not produce remote degeneration of nerve fibers in the nerve trunk or of sensory neurons in the DRG, it was not determined whether capsaicin produced degeneration locally at the site of application. It has been shown, however, that systemic administration of capsaicin caused some degeneration of the subepidermal neural plexus (Chung et al., 1990), demonstrating susceptibility of intracutaneous nerve fibers 

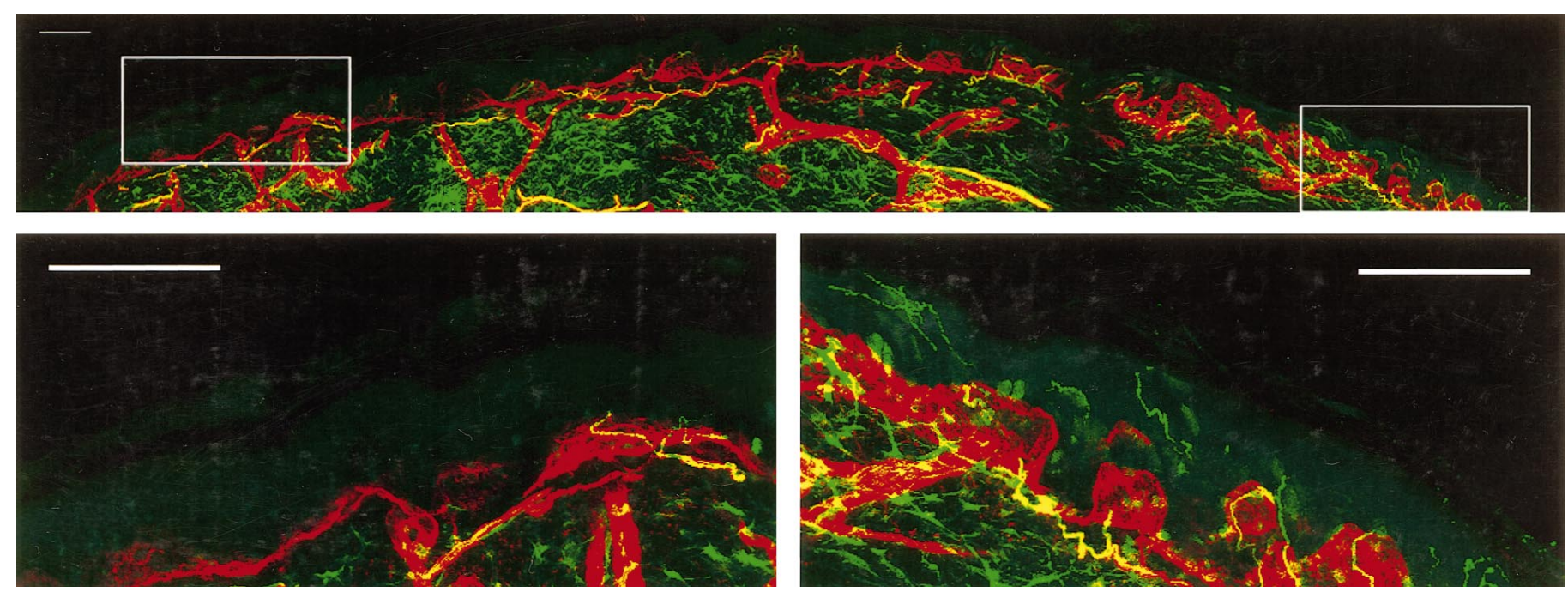

Figure 6. The localization of degeneration of ENFs after an intradermal injection of $20 \mu \mathrm{g}$ of capsaicin as shown by confocal images of skin biopsy from one subject. Top, Montage of confocal images that span across the skin biopsy. Abundant ENFs are seen only at the far right portion of the biopsy, which was located outside the capsaicin injection site and presumably not exposed to the neurotoxin. Bottom, The two opposite ends of the skin biopsy, outlined by the squares in the top image, shown at greater magnification to illustrate differences in epidermal innervation.

to the neurotoxic actions of capsaicin. Also, local application of capsaicin has been shown to cause degeneration of DRG neurons and axons (Handwerker et al., 1984; Marsh et al., 1987; Pini et al., 1990). As illustrated in the present study, degeneration occurred only at the site of capsaicin application and in those fibers exposed to the neurotoxin. Moreover, degeneration was progressive in that only ENFs were affected at $24 \mathrm{hr}$ after capsaicin, whereas degeneration included the subepidermal neural plexus within 1 week and the dermal CGRP- and SP-immunoreactive nerve fibers. This was illustrated more clearly in a parallel study (Nolano et al., 1996) in which repeated topical application of capsaicin produced gradual degeneration of nerve fibers in the epidermis. Recently, Reilly et al. (1997) confirmed the use of the blister technique to show degeneration of ENFs by capsaicin. Thus, capsaicin seems to produce a gradual but limited dying back of fibers from the nerve endings in the epidermis. This pattern of degeneration is common with various types of clinical neuropathies, such as diabetic neuropathy (Kennedy et al., 1996) and neuropathy associated with human immunodeficiency virus injection (McCarthy et al., 1995).

\section{Functions of epidermal nerve fibers}

The present findings provide new information about the function of ENFs. Because loss of ENFs correlated primarily with diminished pain sensation, we believe that many of the ENFs are nociceptors. Furthermore, many are likely to be polymodal nociceptors because pain evoked by heat and mechanical stimuli was depressed. However, an interesting paradox is that cold and tactile sensitivities were not altered significantly, although virtually all fibers in the superficial skin were absent. Although the probe used for cold sensation was small and maintained at very low temperature, it evoked cold sensation without pain. This suggests that cold-specific receptors normally sensitive to innocuous cold temperatures were excited. The finding that cold and tactile sensations were not altered by capsaicin is in agreement with electrophysiological studies showing that evoked responses of low threshold receptors were not altered after intradermal injection of capsaicin (Baumann et al., 1991). It is likely that those sensations arose from activation of receptors located deep in the dermis or just adjacent to the capsaicin injection where innervation is normal. Similarly, deep nociceptors or activated proximal segments of ENFs are likely to account for the residual pricking pain sensation that persisted after capsaicin treatment.

There seemed to be a mismatch in the relationship between the number of regenerated ENFs and evoked sensation. During reinnervation when there were relatively few fibers in the epidermis, there was a striking return of heat and sharp pain sensation. For example, at 3 weeks after capsaicin injection, the mean magnitude of heat pain and sharp pain sensation was 61 and $77 \%$, respectively, of that obtained in normal skin. At this time, however, epidermal reinnervation was only $\sim 17 \%$ of normal. Two possibilities might account for the apparent mismatch between the magnitude of sensation and the number of epidermal nerve fibers. One is that relatively few epidermal nerve fibers are needed for pain detection. This is supported by microneurography studies in humans that suggest activation of a small number of nociceptive primary afferent fibers evoke clear pain sensation (Ochoa and Torebjörk, 1989). If this is true, sensory testing by conventional methods may not be sensitive enough to detect neuropathy in the early stages of degeneration. A second possibility is that the subepidermal neural plexus and receptors located on these fibers contribute to evoked sensation. Indeed at 3 weeks or less after capsaicin treatment, the subepidermal neural plexus appeared to have returned to normal, on visual inspection, with respect to its density and continuity.

\section{Conclusions}

The present study demonstrates that the hypalgesia after application of capsaicin to the skin results from degeneration of ENFs. This finding has important clinical implications because topical capsaicin has been used for a variety of painful syndromes, including diabetic neuropathy. Because we have shown that degeneration of ENFs also occurs after topical capsaicin, although the degeneration has a slower onset and is not as severe as that produced by intradermal injection (Nolano et al., 1996), it is debatable whether capsaicin should be used in syndromes in which there is ongoing nerve pathology and nerve regeneration is necessary to preserve or restore detection of noxious stimuli. In 
this regard, the capsaicin model may be useful to study mechanisms of regeneration of intracutaneous nerve fibers and to assess the effects of neurotrophins and other pharmacological agents in correlative morphological and psychophysical studies.

\section{REFERENCES}

Baumann TK, Simone DA, Shain CN, LaMotte RH (1991) Neurogenic hyperalgesia: the search for the primary afferent nerve fibers that contribute to capsaicin-induced pain and hyperalgesia. J Neurophysiol 66:212-227.

Bevan SJ, Docherty RJ (1993) Cellular mechanisms of the action of capsaicin. In: Capsaicin in the study of pain (Wood J, ed), pp 27-44. London: Academic.

Bleakman D, Brorson JR, Miller RJ (1990) The effect of capsaicin on voltage-gated calcium currents and calcium signals in cultured dorsal root ganglion cells. Br J Pharmacol 101:423-431.

Buck SH, Burks TF (1986) The neuropharmacology of capsaicin: review of some recent observations. Pharmacol Rev 38:179-226.

Capsaicin Study Group (1991) Arch Intern Med 151:2225-2229.

Carpenter SE, Lynn B (1981) Vascular and sensory responses of human skin to mild injury after topical treatment with capsaicin. Br J Pharmacol 73:755-759.

Caterina MJ, Schumacher MA, Tominaga M, Rosen TA, Levine JD, Julius D (1997) The capsaicin receptor: a heat-activated ion channel in the pain pathway. Nature 389:816-824.

Chung K, Klein CM, Coggeshall RE (1990) The receptive part of the primary afferent axon is most vulnerable to systemic capsaicin in adult rats. Brain Res 511:222-226.

Culp WJ, Ochoa JL, Cline M, Dotson R (1989) Heat and mechanical hyperalgesia induced by capsaicin. Cross modality threshold modulation in human C nociceptors. Brain 112:1317-1331.

Docherty RJ, Robertson B, Bevan S (1991) Capsaicin causes prolonged inhibition of voltage-activated calcium currents in adult rat dorsal root ganglion neurons in culture. Neuroscience 40:513-521.

Fitzgerald M (1983) Capsaicin and sensory neurones-a review. Pain 15:109-130.

Fusco BM, Giacovazzo M (1997) Peppers and pain. The promise of capsaicin. Drugs 53:909-914.

Handwerker HO, Holzer-Petsche U, Heym C, Welk E (1984) C-fibre functions after topical application of capsaicin to a peripheral nerve and after neonatal capsaicin treatment. In: Antidromic vasodilatation and neurogenic inflammation (Chahl LA, Szolcsányi J, Lembeck F, eds), pp 57-78. Budapest: Akadémiai Kiadó.

Heyman I, Rang HP (1985) Depolarizing responses to capsaicin in a subpopulation of dorsal root ganglion cells. Neurosci Lett 56:69-75.

Holzer P (1988) Local effector functions of capsaicin-sensitive sensory nerve endings: involvement of tachykinins, calcitonin gene-related peptide and other neuropeptides. Neuroscience 24:739-768.

Holzer P (1991) Capsaicin: cellular targets, mechanisms of action, and selectivity for thin sensory neurons. Pharmacol Rev 43:143-201.

Karanth SS, Pringall DR, Kuhn DM, Levene MM, Polak M (1991) An immunocytochemical study of cutaneous innervation and the distribution of neuropeptides and protein gene product 9.5 in man and commonly employed laboratory animals. Am J Anat 191:369-383.

Kennedy WR, Wendelschafer-Crabb G (1993) The innervation of the human epidermis. J Neurol Sci 115:184-190.

Kennedy WR, Wendelschafer-Crabb G, Johnson T (1996) Quantitation of epidermal nerves in diabetic neuropathy. Neurology 47:1042-1048.

LaMotte RH, Shain CN, Simone DA, Tsai E-F (1991) Neurogenic hyperalgesia: psychophysical studies of underlying mechanisms. J Neurophysiol 66:190-211.

LaMotte RH, Lundberg LER, Torebjörk HE (1992) Pain, hyperalgesia and activity in nociceptive $\mathrm{C}$ units after intradermal injection of capsaicin. J Physiol (Lond) 448:749-764.

Maggi CA, Meli A (1988) The sensory-efferent function of capsaicinsensitive sensory neurons. Gen Pharmacol 19:1-43.

Marsh SJ, Stansfeld CE, Brown DA, Davey R, McCarthy D (1987) The mechanism of action of capsaicin on sensory C-type neurons and their axons in vitro. Neuroscience 23:275-290.

McCarthy BG, Hsieh S-T, Stocks A, Hauer P, Macko C, Cornblath DR, Griffin JW, McArthur JC (1995) Cutaneous innervation in sensory neuropathies: evaluation by skin biopsy. Neurology 45:1848-1855.

McMahon SB, Lewin G, Bloom SR (1991) The consequences of longterm topical capsaicin application in the rat. Pain 44:301-310.

Nagy JI (1982) Capsaicin: a chemical probe for sensory neuron mechanisms. In: Handbook of psychopharmacology, Vol 15 (Iversen LL, Iversen SD, Snyder SH, eds), pp 185-235. New York: Plenum.

Nolano M, Simone DA, Wendelschafer-Crabb G, Kennedy WR (1996) Decreased sensation and loss of epidermal nerve fibers following repeated topical application of capsaicin in humans. Soc Neurosci Abstr 22:1802.

Ochoa JL (1984) Peripheral unmyelinated units in man: structure, function, disorder, and role in sensation. In: Advances in pain research and therapy, Vol 6 (Kruger L, Liebeskind JC, eds), pp 53-68. New York: Raven.

Ochoa JL, Torebjörk H (1989) Sensations evoked by intraneural microstimulation of $\mathrm{C}$ nociceptor fibres in human skin nerves. J Physiol (Lond) 415:583-599.

Pini A, Baranowski R, Lynn B (1990) Long-term reduction in the number of C-fibre nociceptors following capsaicin treatment of a cutaneous nerve in adult rats. Eur J Neurosci 2:89-97.

Reilly DM, Ferdinando D, Johnston C, Shaw C, Buchanan KD, Green MR (1997) The epidermal nerve fibre network: characterization of nerve fibres in human skin by confocal microscopy and assessment of racial variations. Br J Dermatol 137:163-170.

Russell LC, Burchiel KJ (1984) Neurophysiological effects of capsaicin. Brain Res Rev 8:165-176.

Saria A, Martling CR, Yan Z, Theodorsson-Norheim E, Gamse R, Lundberg JM (1988) Release of multiple tachykinins from capsaicinsensitive sensory nerves in the lung by bradykinin, histamine, dimethylphenyl piperazinium, and vagal nerve stimulation. Am Rev Respir Dis 137:1330-1335.

Simone DA, Ochoa JL (1991) Early and late effects of prolonged topical capsaicin on cutaneous sensibility and neurogenic vasodilatation in humans. Pain 47:285-293.

Simone DA, Ngeow JYF, Putterman GJ, LaMotte RH (1987) Hyperalgesia to heat after intradermal injection of capsaicin. Brain Res 418:201-203.

Simone DA, Baumann TK, LaMotte RH (1989) Dose-dependent pain and mechanical hyperalgesia in humans after intradermal injection of capsaicin. Pain 38:99-107.

Simone DA, Nolano M, Wendelschafer-Crabb G, Kennedy WR (1996) Intradermal injection of capsaicin in humans: diminished pain sensation associated with rapid degeneration of intracutaneous nerve fibers. Soc Neurosci Abstr 22:1802.

Szallasi A, Blumberg PM (1990a) Specific binding of resiniferatoxin, an ultrapotent capsaicin analog, by dorsal root ganglion membranes. Brain Res 524:106-111.

Szallasi A, Blumberg PM (1990b) Resiniferatoxin and its analogs provide novel insights into the pharmacology of the vanilloid (capsaicin) receptor. Life Sci 47:1399-1408.

Szolcsányi J (1977) A pharmacological approach to elucidation of the role of different nerve fibres and receptor endings in mediation of pain. J Physiol (Paris) 73:251-259.

Szolcsányi J (1993) Actions of capsaicin on sensory receptors. In: Capsaicin in the study of pain (Wood J, ed), pp 1-26. London: Academic.

Szolcsányi J, Janscó-Gábor A, Joó F (1975) Functional and fine structural characteristics of the sensory neuron blocking effect of capsaicin. Naunyn Schmiedebergs Arch Pharmacol 287:157-169.

Wang L, Hilliges M, Jernberg T, Wiegleb-Edström D, Johansson O (1990) Protein gene product 9.5-immunoreactive nerve fibres and cells in human skin. Cell Tissue Res 261:25-33.

Wood JN, Winter J, James IF, Rang HP, Yeats J, Bevan S (1988) Capsaicin-induced ion fluxes in dorsal root ganglion cells in culture. J Neurosci 8:3208-3220. 